\title{
Wave envelopes with second-order spatiotemporal dispersion: II. Modulational instabilities and dark Kerr solitons
}

\author{
J. M. Christian, G. S. McDonald, and T. F. Hodgkinson \\ Joule Physics Laboratory, School of Computing, Science and Engineering, \\ Materials \& Physics Research Centre, University of Salford, Greater Manchester M5 4WT, United Kingdom

\section{P. Chamorro-Posada} \\ Departamento de Teoría de la Señal y Comunicaciones e Ingeniería Telemática, \\ Universidad de Valladolid, ETSI Telecomunicación, Campus Miguel Delibes Paseo Belén 15, \\ E-47011 Valladolid, Spain
}

\section{$\left(3^{\text {th }}\right.$ March 2012 - submitted to Physical Review A)}

\begin{abstract}
A simple scalar model for describing spatiotemporal dispersion of pulses, beyond the classic "slowly-varying envelopes + Galilean boost" approach, is studied. The governing equation has a cubic nonlinearity and we focus here mainly on contexts with normal group-velocity dispersion. A complete analysis of continuous waves is reported, including their dispersion relations and modulational instability characteristics. We also present a detailed derivation of exact analytical dark solitons, obtained by combining direct-integration methods with geometrical transformations. Classic results from conventional pulse theory are recovered asymptotically from the spatiotemporal formulation. Numerical simulations test new theoretical predictions for modulational instability, and examine the robustness of spatiotemporal dark solitons against perturbations to their local pulse shape.
\end{abstract}

PACS numbers: 42.65.- $\mathrm{k}$ (nonlinear optics),

42.65.Tg (optical solitons),

42.25.-p (wave optics),

05.45.Yv (solitons, nonlinear dynamics of). 


\section{INTRODUCTION}

When modelling wave phenomena in physical systems, one is typically concerned with describing the evolution of a real quantity $Q(t, z)$ that may be represented as an envelope $q(t, z)$ modulating a rapidlyoscillating component according to $Q(t, z)=q(t, z) \exp \left[i\left(k_{0} z-\omega_{0} t\right)\right]+q^{*}(t, z) \exp \left[-i\left(k_{0} z-\omega_{0} t\right)\right]$. Here, $t$ and $z$ denote time and space coordinates in the laboratory frame, respectively, while $k_{0}$ and $\omega_{0}$ are the propagation constant and angular frequency of the underlying carrier wave. The variable $Q$ may correspond to elastic displacement, electric field, polarization grating, fluid velocity, ion density, quantum mechanical wavefunction, etc. [1-3]. The slowly-varying envelope approximation (SVEA), which anticipates that the longitudinal variation of $q$ is slow on the $\sim 1 / k_{0}$ scale-length, is the first part of near-universal mathematical device whose function is to reduce complicated governing equations to a more tractable structure. The second part is the deployment of a Galilean-type coordinate boost to a frame of reference $\left(t_{\mathrm{loc}}, z_{\mathrm{loc}}\right)$ moving at some characteristic (system dependent) speed, typically the group velocity $v_{g}$. Such a transformation has the standard form $t_{\mathrm{loc}} \equiv t-z / v_{g}$ and $z_{\mathrm{loc}} \equiv z$ (the local time frame is denoted throughout by the "loc" subscript).

The "SVEA + Galilean boost" recipe is used so freely in the literature that one rarely queries its adoption. One of the key advantages of this classic approach is that it can operate largely independently of system nonlinearity, and it is certainly true that the technique clearly works well in the vast majority of studies to date. However, an inevitable consequence is that relatively little is known about the mathematical properties of the more general governing equations and the properties (e.g., structure and stability) of its solutions. One should also be mindful that there are classes of problem where such a conventional modelling approach is not necessarily appropriate.

We investigate a dimensionless universal spatiotemporal dispersion model having cubic nonlinearity $[4,5]$, with particular emphasis on the case of normal group-velocity dispersion. This generic equation, which for example, arises in photonics and waveguide optics [6], is of the form

$$
\kappa \frac{\partial^{2} u}{\partial \zeta^{2}}+i\left(\frac{\partial u}{\partial \zeta}+\alpha \frac{\partial u}{\partial \tau}\right)+\frac{s}{2} \frac{\partial^{2} u}{\partial \tau^{2}}+|u|^{2} u=0
$$

where $u$ is the wave envelope, $\zeta$ denotes the (longitudinal) space coordinate along which the wavepacket is travelling, and $\tau$ denotes time (note that $\tau$ does not represent a local time variable). The three parameters appearing in Eq. (1) are: $\alpha$ (proportional to a ratio of group speeds), $s= \pm 1$ [flagging the group-velocity dispersion (GVD) regime: +1 for anomalous; -1 for normal] and $\kappa$ (parameterizing the strength of spatial dispersion). Spatial and temporal dispersion phenomena are thus identified with the $\kappa \partial^{2} / \partial \zeta^{2}$ and $(s / 2) \partial^{2} / \partial \tau^{2}$ operators, respectively. The mathematical structure of Eq. (1) depends upon the product $s \kappa$ [for $\operatorname{sgn}(s \kappa)=+1(-1)$, it is elliptic (hyperbolic) in character].

The reader is directed to the Appendix for a derivation of model (1) in an optical context where, historically, the "SVEA + Galilean boost" has often been applied [7,8]. This latter approach usually works well provided the pulses under consideration are relatively long (where a sufficient number of electric-field cycles are contained within the envelope) and one is operating sufficiently far from the zero-dispersion point [2,611]. When such conditions are not met, one may choose to accommodate higher-order linear effects through 
a succession of additional terms of the type $\gamma_{j}(i \partial / \partial \tau)^{j} u$ (where $j=3,4,5 \ldots$ and the $\left\{\gamma_{j}\right\}$ are real parameters) [12], or abandon the notion of an "envelope" altogether and instead work with the short-pulse equation [13]. Recently, it has been shown that there exist some semiconductor materials (e.g., ZnCdSe / ZnSe superlattices) where the role played by the $\kappa \partial^{2} / \partial \zeta^{2}$ operator is no longer marginal and cannot be neglected in the traditional way [14]. A striking feature of spatial dispersion (an effect allied to $\kappa \partial^{2} u / \partial \zeta^{2}$ ) is that the parameter $\kappa$ in Eq. (1) may assume either sign.

In Ref. 5, the transformation and conservation laws were derived for Eq. (1). Exact analytical bright solitons were found to exist when $s=+1$, along with two classes of temporally-extended solutions (cnoidal and dnoidal waves, which describe periodic trains of pulses). Two distinct, but equivalent, solution representations were also developed. Extensive computations, in parallel with classic analytical methods [15], confirmed the bright soliton pulses as highly robust entities surrounded by wide basins of attraction.

Here, we complete the analysis of model (1). The focus is predominantly on exact analytical dark solitons, which exist when $s=-1$ and comprise a continuous-wave (cw) background field modulated by a phasetopological grey "dip." The stability of the cw background is an issue of fundamental importance since these solutions play a key role in dark-soliton structure [16]. Our motivation is to establish the stability properties of the dark solitons of Eq. (1), and to see whether they exhibit a similar degree of robustness to their bright counterparts.

The layout of this paper is as follows. In Sec. II, we review the mathematical steps taken to obtain the conventional pulse model from Eq. (1). In Sec. III, the cw solutions to Eq. (1) are derived, their dispersion relations are characterized, and the subtle recovery of the corresponding conventional solutions is demonstrated. Linearization techniques are then deployed to investigate the resilience of $\mathrm{cw}$ solutions to small perturbative modulations, and both long- and short-wave instability regimes are identified. Subsequent numerical computations validate theoretical predictions. In Sec. IV, we report exact analytical dark solitons (black and more general grey families), and also a class of snoidal wave (essentially a black-soliton pulse train). The space-time structure of these more exotic solutions is detailed, and the recovery of their conventional counterparts, is described in Sec. V. Sets of simulations, testing the robustness of dark solitons against perturbations to their temporal shape, are presented in Sec. VI. Geometrical transformations are used to predict asymptotic pulse parameters. We conclude, in Sec. VII, with some comments on the significance and potential applications of these new results.

\section{SLOWLY-VARYING ENVELOPES AND GALILEAN BOOSTS}

Conventional pulse theory makes an initial assumption that the first term in Eq. (1) may be neglected. Adoption of the SVEA, which is embodied by the inequality $\left|\kappa \partial^{2} u / \partial \zeta^{2}\right| \ll|\partial u / \partial \zeta|$, reduces the governing equation to the more tractable form 


$$
i\left(\frac{\partial u}{\partial \zeta}+\alpha \frac{\partial u}{\partial \tau}\right)+\frac{s}{2} \frac{\partial^{2} u}{\partial \tau^{2}}+|u|^{2} u \simeq 0
$$

One then condenses the differential-operator combination $\partial / \partial \zeta+\alpha \partial / \partial \tau$ into a single derivative by introducing local coordinates, $\tau_{\mathrm{loc}} \equiv \tau-\alpha \zeta$ and $\zeta_{\text {loc }} \equiv \zeta$. Under this Galilean-type boost to a frame of reference moving at speed $1 / \alpha$ in the $+\zeta$ direction, $\partial / \partial \zeta$ and $\partial / \partial \tau$ transform individually as $\partial / \partial \zeta=\partial / \partial \zeta_{\text {loc }}-\alpha \partial / \partial \tau_{\text {loc }}$ and $\partial / \partial \tau=\partial / \partial \tau_{\text {loc }}$, combining to leave a parabolic wave operator in the local time frame: $i(\partial / \partial \zeta+\alpha \partial / \partial \tau)+(s / 2) \partial^{2} / \partial \tau^{2}=i \partial / \partial \zeta_{\mathrm{loc}}+(s / 2) \partial^{2} / \partial \tau_{\mathrm{loc}}^{2}$ [7-11]. Since the nonlinearity is left unchanged under boost transformation (this is true even when the nonlinearity involves time dependence, e.g., for Raman-type contributions [17]), the solution $u\left(\tau_{\mathrm{loc}}, \zeta_{\mathrm{loc}}\right)$ satisfies the canonical nonlinear Schrödinger (NLS) equation, namely $\left[i \partial / \partial \zeta_{\mathrm{loc}}+(s / 2) \partial^{2} / \partial \tau_{\mathrm{loc}}^{2}+|u|^{2}\right] u\left(\tau_{\mathrm{loc}}, \zeta_{\mathrm{loc}}\right) \simeq 0$.

This standard approach is unhelpful if, for instance, one wishes to retain the $\kappa \partial^{2} u / \partial \zeta^{2}$ term [4$6,14,17,18]$. By abandoning the Galilean boost and remaining in the laboratory frame, Eq. (1) can instead be analysed within a more exact framework (see Appendix B) involving space-time coordinate transformations that are remarkably similar to those in Einstein's special theory of relativity. It is then found that the predictions of conventional pulse theory [i.e., Eq. (2)] tend to be recovered asymptotically from the more general model [i.e., Eq. (1)] in the same formal way that Newtonian mechanics emerge in the low-speed limit of relativistic mechanics.

\section{CONTINUOUS WAVES AND MODULATIONAL INSTABILITY}

\section{A. Dispersion relations}

The cw solutions of Eq. (1) have the form $u(\tau, \zeta)=\rho_{0}{ }^{1 / 2} \exp [i(-\Omega \tau+K \zeta)] \exp (-i \zeta / 2 \kappa)$, where $\rho_{0} \equiv|u|^{2}$ is the wave intensity, $\Omega$ measures the (normalized) deviation of the envelope from the carrier-wave frequency, and $K$ is the propagation constant. Substitution of $u$ into Eq. (1) yields the quadratic dispersion relation

$$
\kappa K^{2}-\frac{1}{4 \kappa}-\Omega\left(\alpha-\frac{1}{2} s \Omega\right)=\beta,
$$

where we have introduced $\beta \equiv \rho_{0}$ (the reasons for this choice will become clear shortly). When $\operatorname{sgn}(s \kappa)=$ $+1(-1)$, Eq. (3a) prescribes families of ellipses (hyperbole) in the $(\Omega, K)$ plane that are parameterized by $\kappa$, $s, \alpha$ and $\beta$. The corresponding cw solutions are then given by [4]

$$
\begin{aligned}
u(\tau, \zeta) & =\rho_{0}^{1 / 2} \exp (-i \Omega \tau) \\
& \times \exp \left[ \pm i \sqrt{1+4 \kappa \beta+4 \kappa \Omega\left(\alpha-\frac{1}{2} s \Omega\right)} \frac{\zeta}{2 \kappa}\right] \\
& \times \exp \left(-i \frac{\zeta}{2 \kappa}\right),
\end{aligned}
$$

where the \pm sign denotes propagation in the forward and backward longitudinal directions, respectively. For $\operatorname{sgn}(s \kappa)=+1$ (ellipses), non-evanescent waves exist within the (displaced) frequency band $\Omega<\Omega<\Omega_{+}$, 
where $\Omega_{ \pm}=s \alpha \pm\left[\alpha^{2}+(2 s \beta+1 / 2|s \kappa|)\right]^{1 / 2}$. For $\operatorname{sgn}(s \kappa)=-1$ (hyperbole), there are no such propagation cutoffs (see Fig. 1).

\section{B. Recovery of conventional waves}

When all contributions from the $\kappa \partial^{2} u / \partial \zeta^{2}$ term are negligibly small simultaneously, one must be able to recover the predictions made by conventional pulse theory. For the cw solutions of Eq. (1), the three-fold algebraic limit $\kappa \rightarrow 0$ (negligible spatial dispersion), $\kappa \beta \rightarrow 0$ (negligible nonlinear phase shift), and $\kappa \Omega[\alpha-$ $(s / 2) \Omega] \rightarrow 0$ (negligible frequency shift) is formally equivalent to the SVEA. The forward wave in Eq. (3b) converges to $u(\tau, \zeta) \simeq \rho_{0}{ }^{1 / 2} \exp \left[-i \Omega(\tau-\alpha \zeta)+i\left(\beta-s \Omega^{2} / 2\right) \zeta\right]$ so that, in the local time frame, the solution $u\left(\tau_{\mathrm{loc}}, \zeta_{\mathrm{loc}}\right) \simeq \rho_{0}{ }^{1 / 2} \exp \left[-i \Omega \tau_{\mathrm{loc}}+i\left(\beta-s \Omega^{2} / 2\right) \zeta_{\mathrm{loc}}\right]$ satisfies the canonical NLS equation of Sec II. Thus, the parameter $\beta$ can be identified with the propagation constant of the corresponding $\Omega=0$ conventional wave.

The same algebraic procedure may also be applied to the backward wave in Eq. (3b), whereupon it is found that $u(\tau, \zeta) \simeq \rho_{0}{ }^{1 / 2} \exp \left[-i \Omega(\tau+\alpha \zeta)-i\left(\beta-s \Omega^{2} / 2\right) \zeta\right] \exp [-i 2(\zeta / 2 \kappa)]$. While boosting to a local time frame moving in the backward direction is possible (e.g., by introducing $\tau_{\text {loc }} \equiv \tau+\alpha \zeta$ and $\zeta_{\text {loc }} \equiv \zeta$ ), there is no advantage in so-doing: this transformed wave $u\left(\tau_{\text {loc }}, \zeta_{\text {loc }}\right)$ clearly cannot satisfy the approximate equation $\left[i\left(\partial / \partial \zeta_{\mathrm{loc}}+2 \alpha \partial / \partial \tau_{\mathrm{loc}}\right)+(s / 2) \partial^{2} / \partial \tau_{\mathrm{loc}}^{2}+|u|^{2}\right] u\left(\tau_{\mathrm{loc}}, \zeta_{\mathrm{loc}}\right) \simeq 0$ because of the $\kappa$-dependent rapid phase factor that survives the limit process. The conventional model is inherently uni-directional (describing evolution with respect to a single longitudinal direction) and does not support backward propagation.

\section{Linear stability analysis}

Attention is now turned to the stability of the cw solutions against small-amplitude modulations in the time domain. Without loss of generality, we set $\Omega=0$ and express $u$ as $u(\tau, \zeta)=\rho_{0}{ }^{1 / 2}[1+\varepsilon a(\tau, \zeta)] \exp (i K \zeta)$ $\exp (-i \zeta / 2 \kappa)$, where $\varepsilon$ is a formal expansion parameter and $a(\tau, \zeta)$ is a complex function that describes a disturbance to the amplitude and phase of the stationary state. By considering small modulations, where $|\varepsilon| \ll$ $O(1)$ and $|a|=O(1)$, one can linearize in $a$ around the cw solution and derive the following equation at $O(\varepsilon)$ :

$$
\kappa \frac{\partial^{2} a}{\partial \zeta^{2}} \pm i \sqrt{1+4 \kappa \beta} \frac{\partial a}{\partial \zeta}+i \alpha \frac{\partial a}{\partial \tau}+\frac{s}{2} \frac{\partial^{2} a}{\partial \tau^{2}}+\rho_{0}\left(a+a^{*}\right)=0 .
$$

The simplest perturbation to analyse is a single Fourier component with frequency shift $\Omega_{p}$. Such a component can be represented by

$$
a(\tau, \zeta)=a_{1} \exp \left(-i \Omega_{p} \tau+i K_{p} \zeta\right)+a_{2}^{*} \exp \left(+i \Omega_{p} \tau-i K_{p}^{*} \zeta\right)
$$

where $a_{1}$ and $a_{2}$ are arbitrary constants. Here, $K_{p} \equiv k_{p}+i \Lambda_{p}$ is a complex wavenumber whose real and imaginary parts correspond to the propagation constant and spatial growth rate of Fourier mode (5), respectively.

After substituting $a(\tau, \zeta)$ into Eq. (4), one arrives at the following characteristic equation for $K_{p}$ :

$$
\begin{aligned}
& \kappa^{2} K_{p}^{4}-\left(1+6 \kappa \beta-s \Omega_{p}^{2}\right) K_{p}^{2}+2 \alpha \Omega_{p} \sqrt{1+4 \kappa \beta} K_{p} \\
& +\frac{1}{2} \Omega_{p}^{2}\left[\frac{1}{2} \Omega_{p}^{2}-2\left(\alpha^{2}+s \rho_{0}\right)\right]=0 .
\end{aligned}
$$


The third term in Eq. (6), which arises from the $i \alpha \partial / \partial \tau$ operator, has no counterpart in the corresponding spatial calculation [19]. This linear-in- $K_{p}$ factor frustrates subsequent algebraic analysis because one cannot first solve for $K_{p}^{2}$. It is possible, in principle, to solve Eq. (6) for $K_{p}$ directly with recourse to the standard formula for finding the roots of quartic equations [20]. However, these solutions are algebraically complex and provided little physical insight into the stability problem.

\section{Long-wave and short-wave instabilities}

We restrict our attention to regimes with $\beta=O(1)$ and where there is only a small level of spatial dispersion, $|\kappa| \ll O(1)$. When $\operatorname{sgn}(\kappa s)=+1$ (see Fig. 2$), \operatorname{Re}\left[K_{p}\left(\Omega_{p}\right)\right]$ comprises a pair of ellipses of approximate width $(2 /|\kappa|)^{1 / 2}$ centred on $\Omega_{p} \simeq \pm \alpha$, while $\operatorname{Im}\left[K_{p}\left(\Omega_{p}\right)\right]$ comprises a set of four hyperbole that characterize a short-wave instability. There also exists a long-wave instability near the origin $\Omega_{p} \simeq 0$ [see inset of Fig. 2(b)]. In this domain, where $\kappa K_{p}{ }^{2} \rightarrow 0, \kappa \Omega_{p}{ }^{2} \rightarrow 0$, and $\kappa \beta \rightarrow 0$, Eq. (6) is well approximated by the parabola

$$
K_{p}^{2}-2 \alpha \Omega_{p} K_{p}-\frac{1}{2} \Omega_{p}^{2}\left[\frac{1}{2} \Omega_{p}^{2}-2\left(\alpha^{2}+s \rho_{0}\right)\right] \simeq 0,
$$

which can be solved immediately to yield

$$
K_{p}=\alpha \Omega_{p} \pm \sqrt{\frac{1}{2} \Omega_{p}^{2}}\left(\frac{1}{2} \Omega_{p}^{2}-2 s \rho_{0}\right)^{1 / 2} .
$$

For $s=+1$, modulational instability (MI) appears in the frequency band $\left|\Omega_{p}\right|<\left(4 \rho_{0}\right)^{1 / 2}$, where $K_{p}$ acquires a non-zero imaginary component. The most unstable frequency is $\Omega_{p 0}=\left(2 \rho_{0}\right)^{1 / 2}$, which is associated with a period $T_{p 0} \equiv 2 \pi / \Omega_{p 0}=2^{1 / 2} \pi / \rho_{0}{ }^{1 / 2}$. This long-wave instability vanishes for $s=-1$ [irrespective of $\operatorname{sgn}(\kappa)$ ] because $K_{p}$ is always real. It is interesting to note that Eqs. (7a) and (7b) coincide with the predictions of conventional pulse theory. After a Galilean boost to the local time frame, the first term on the right-hand side of Eq. (7b) is transformed away and one is left with the classic result $K_{p}\left(\Omega_{p}\right)= \pm\left|\Omega_{p}\right|\left(\Omega_{p}{ }^{2} / 4-s \rho_{0}\right)^{1 / 2}[21]$. When $\operatorname{sgn}(s \kappa)=-1, \operatorname{Re}\left(K_{p}\right)$ always comprises four hyperbole. For $s=+1$ and $\kappa<0, \operatorname{Im}\left[K_{p}\left(\Omega_{p}\right)\right]$ exhibits only the long-wave MI band [see Fig. 2(b)]; when $s=-1$ and $\kappa>0$, one finds $\operatorname{Im}\left(K_{p}\right)=0$ (i.e., no MI at any $\left.\Omega_{p}\right)$.

\section{E. Spontaneous modulational instability}

Analysis predicts, and simulations confirm, that there is no growth of long-wave instability when $\rho_{0}=0$; indeed, bright solitons (i.e., pulses on a zero-amplitude cw background) were found to behave like robust attractors [5]. Results are now presented from simulations of model (1) with $s=+1$. A cw solution with $\rho_{0}=$ $O(1)$ and $\Omega=0$ is initialized and a $0.01 \%$ level of complex noise is added to accelerate the growth of any spontaneous instability. During the early stages of evolution, before fully-developed nonlinear dynamics take hold of the system, long-wave sidebands corresponding to the most-unstable frequency [predicted by Eq. (7b)] grow first (see Fig. 3). Simulations have not uncovered any evidence of long-wave instabilities in regimes with $s=-1$, providing numerical confirmation of our analytical predictions. 


\section{DARK SOLITON PULSES}

In this Section, two equivalent representations of the exact analytical dark solitons of Eq. (1) are derived. One expects families of tanh-type solutions to exist in the normal-GVD regime, where $s=-1$. However, these particular solution classes are much more difficult to derive than their bright counterparts [5]. A direct-integration method is developed for the governing equation, in which the dark solution $u$ is explicitly decomposed into its cw background field and grey-dip (intensity and phase) quadratures. A range of coordinate transformation techniques is then deployed to arrive at a more general dark solution [22].

\section{A. Coordinate transformation laws and}

\section{velocity combination rule}

It is worthwhile to review some essential mathematical results that will be used extensively throughout the following derivation and analysis. Under the coordinate change

$$
\tau=\frac{\tau^{\prime}-V \zeta^{\prime}}{\sqrt{1+2 s \kappa V^{2}}} \quad \text { and } \quad \zeta=\frac{2 s \kappa V \tau^{\prime}+\zeta^{\prime}}{\sqrt{1+2 s \kappa V^{2}}}
$$

where $V$ is a velocity-like parameter, the form invariance of Eq. (1) is preserved so long as $u$ transforms according to

$$
\begin{aligned}
u(\tau, \zeta) & =\exp \left[-i \frac{s V \tau^{\prime}}{\sqrt{1+2 s \kappa V^{2}}}\right. \\
& \left.+\frac{i}{2 \kappa}\left(1-\frac{1}{\sqrt{1+2 s \kappa V^{2}}}\right) \zeta^{\prime}\right] \\
& \times \exp \left[-i s \alpha \frac{\tau^{\prime}-V \zeta^{\prime}}{\sqrt{1+2 s \kappa V^{2}}}+i s \alpha \tau^{\prime}\right] u^{\prime}\left(\tau^{\prime}, \zeta^{\prime}\right) .
\end{aligned}
$$

From Eqs. (8a) and (8b), it can be shown that two arbitrary velocities, denoted by $V_{0}$ and $V$, combine geometrically (not additively, as they do in conventional pulse theory) to give a net velocity $W$, where $[4,5,23]$

$$
W=\frac{V_{0}+V}{1-2 s \kappa V_{0} V} .
$$

This result for $W$ is generic in nature, in the sense that it is independent of both the system nonlinearity and also the solution $u$ being transformed. The structure of Eq. (8d) also bears striking similarity to the velocity combination rule of relativistic kinematics [24]. For brevity, we refer throughout to $V, V_{0}$ and $W$ as background, intrinsic and net velocities, respectively, while remaining mindful of the fact that they are strictly related to inverse velocities in the unscaled laboratory frame.

\section{B. Symmetry reduction and derivation of quadrature equations}

The analysis begins by seeking solutions of the form $u(\tau, \zeta)=\rho^{1 / 2}(\tau, \zeta) \exp [i \Phi(\tau, \zeta)] \exp (-i \zeta / 2 \kappa)$, where $\rho(\tau, \zeta) \equiv|u(\tau, \zeta)|^{2}$ is the (real) wave intensity, $\Phi(\tau, \zeta)$ is the phase distribution, and the complex-exponential $\exp (-i \zeta / 2 \kappa)$ is a manifestation of the underlying carrier wave (this contribution always appears explicitly in 
Helmholtz-type envelope solutions) [25]. Substitution of $u$ into Eq. (1) and isolating the real and imaginary parts, respectively, yields:

$$
\begin{aligned}
& \frac{2}{\rho}\left(\frac{\partial^{2} \rho}{\partial \tau^{2}}-2 \kappa \frac{\partial^{2} \rho}{\partial \zeta^{2}}\right)-\frac{1}{\rho^{2}}\left[\left(\frac{\partial \rho}{\partial \tau}\right)^{2}-2 \kappa\left(\frac{\partial \rho}{\partial \zeta}\right)^{2}\right] \\
& -4\left[\left(\frac{\partial \Phi}{\partial \tau}\right)^{2}-2 \kappa\left(\frac{\partial \Phi}{\partial \zeta}\right)^{2}\right]+8\left(\alpha \frac{\partial \Phi}{\partial \tau}-\frac{1}{4 \kappa}-\rho\right)=0,
\end{aligned}
$$

and

$$
\rho\left(\frac{\partial^{2} \Phi}{\partial \tau^{2}}-2 \kappa \frac{\partial^{2} \Phi}{\partial \zeta^{2}}\right)+\left(\frac{\partial \rho}{\partial \tau} \frac{\partial \Phi}{\partial \tau}-2 \kappa \frac{\partial \rho}{\partial \zeta} \frac{\partial \Phi}{\partial \zeta}\right)-\alpha \frac{\partial \rho}{\partial \tau}=0 .
$$

Physically, dark solitons comprise a grey dip in the intensity profile (described by $\rho$ ) modulating a continuous wave [see Eq. (3b)]. This simplifies the mathematical problem because one may proceed by looking for particular solutions of Eqs. (9a) and (9b) in which $\Phi$ may be expressed as $\Phi(\tau, \zeta)=\Psi(\tau, \zeta)+K \zeta$. Here, $\Psi(\tau, \zeta)$ represents the phase variation across the dark soliton, $K= \pm(1+4 \kappa \beta)^{1 / 2} / 2 \kappa$ [from Eq. (3a), where $\beta$ $\left.\equiv \rho_{0}\right]$ and it has been assumed, for simplicity, that the cw background field has $\Omega=0$ (this restriction will be lifted shortly). The partial derivatives $\partial \Phi / \partial \tau$ and $\partial \Phi / \partial \zeta$ may then be replaced by $\partial \Psi / \partial \tau$ and $\partial \Phi / \partial \zeta+K$, respectively, so that Eqs. (9a) and (9b) become

$$
\begin{aligned}
& \frac{2}{\rho}\left(\frac{\partial^{2} \rho}{\partial \tau^{2}}-2 \kappa \frac{\partial^{2} \rho}{\partial \zeta^{2}}\right)-\frac{1}{\rho^{2}}\left[\left(\frac{\partial \rho}{\partial \tau}\right)^{2}-2 \kappa\left(\frac{\partial \rho}{\partial \zeta}\right)^{2}\right] \\
& -4\left[\left(\frac{\partial \Psi}{\partial \tau}\right)^{2}-2 \kappa\left(\frac{\partial \Psi}{\partial \zeta}\right)^{2}\right] \\
& +8\left(2 \kappa K \frac{\partial \Psi}{\partial \tau}+\alpha \frac{\partial \Psi}{\partial \tau}+\beta-\rho\right)=0
\end{aligned}
$$

and

$$
\begin{aligned}
& \rho\left(\frac{\partial^{2} \Psi}{\partial \tau^{2}}-2 \kappa \frac{\partial^{2} \Psi}{\partial \zeta^{2}}\right)+\left(\frac{\partial \rho}{\partial \tau} \frac{\partial \Psi}{\partial \tau}-2 \kappa \frac{\partial \rho}{\partial \zeta} \frac{\partial \Psi}{\partial \zeta}\right) \\
& -\left(2 \kappa K \frac{\partial \rho}{\partial \zeta}+\alpha \frac{\partial \rho}{\partial \tau}\right)=0 .
\end{aligned}
$$

In this way, the cw component has been eliminated from the problem and one can concentrate solely on deriving the grey-dip part of the solution $u$.

Referring to coordinate change (8), it is convenient to introduce a new coordinate $\xi \equiv\left(\tau-V_{0} \zeta\right) /(1-$ $\left.2 \kappa V_{0}^{2}\right)^{1 / 2}$, where $\tau-V_{0} \zeta=0$ defines the trajectory in the space-time plane along which the pulse centre travels (alternatively, $\xi$ may be interpreted as a rest-frame coordinate). Here, $V_{0}$ is the intrinsic velocity of the grey dip in the (normalized) laboratory frame; for a pulse moving forwards (backwards) along the longitudinal $\zeta$ axis, $V_{0}$ must be positive (negative). The operators $\partial / \partial \tau$ and $\partial / \partial \zeta$ transform as $\partial / \partial \tau=\left(1-2 \kappa V_{0}^{2}\right)^{-1 / 2}(d / d \xi)$ and $\partial / \partial \zeta=-V_{0}\left(1-2 \kappa V_{0}^{2}\right)^{-1 / 2}(d / d \xi)$, while $\partial^{2} / \partial \tau^{2}=\left(1-2 \kappa V_{0}^{2}\right)^{-1}\left(d^{2} / d \xi^{2}\right)$ 
and $\partial^{2} / \partial \zeta^{2}=V_{0}^{2}\left(1-2 \kappa V_{0}^{2}\right)^{-1}\left(d^{2} / d \xi^{2}\right)$. This symmetry reduction approach has thus transformed Eqs. (10a) and (10b) into a pair of coupled ordinary differential equations for the $\rho$ and $\Psi$ quadratures:

$$
\begin{aligned}
\frac{d}{d \rho}\left[\frac{1}{\rho}\left(\frac{d \rho}{d \xi}\right)^{2}\right] & =4\left(\frac{d \Psi}{d \xi}\right)^{2}-8\left(\frac{\alpha-2 \kappa K V_{0}}{\sqrt{1-2 \kappa V_{0}^{2}}}\right) \frac{d \Psi}{d \xi} \\
& -8(\beta-\rho),
\end{aligned}
$$

and

$$
\frac{d}{d \xi}\left(\rho \frac{d \Psi}{d \xi}-\frac{\alpha-2 \kappa K V_{0}}{\sqrt{1-2 \kappa V_{0}^{2}}} \rho\right)=0,
$$

which may be decoupled and solved exactly. Indefinite integration (with respect to $\xi$ ) of Eq. (11b) yields $d \Psi / d \xi=\left(\alpha-2 \kappa K V_{0}\right)\left(1-2 \kappa V_{0}^{2}\right)^{-1 / 2}+c_{1} / \rho$, where $c_{1}$ is a constant to be determined later (by applying solution boundary conditions). The phase slope $d \Psi / d \xi$ can now be eliminated from Eq. (11a), and a first integration (with respect to $\rho$ ) gives

$$
\begin{aligned}
\left(\frac{d \rho}{d \xi}\right)^{2}= & -4 c_{1}^{2}-4 \frac{\left(\alpha-2 \kappa K V_{0}\right)^{2}}{1-2 \kappa V_{0}^{2}} \rho^{2} \\
& -8\left(\beta \rho^{2}-\frac{\rho^{3}}{2}\right)+c_{2} \rho,
\end{aligned}
$$

where $c_{2}$ is a second constant to be determined. The relevant boundary conditions on $\rho$ and $\Psi$ for a grey soliton are:

$$
\lim _{\xi \rightarrow 0} \rho(\xi)=\rho_{1}, \quad \lim _{\xi \rightarrow 0} \frac{d}{d \xi} \rho(\xi)=0, \quad \lim _{\xi \rightarrow \pm \infty} \rho(\xi)=\rho_{0},
$$

where $\rho_{0}$ corresponds to the intensity of the cw background field and $0<\rho_{1} \leq \rho_{0}$ is the intensity at the pulse centre. For dark-type solutions, the $\xi$ derivatives of $\rho$ and $\Psi$ must satisfy vanishing asymptotics:

$$
\lim _{\xi \rightarrow \pm \infty} \frac{d}{d \xi} \rho(\xi)=0, \quad \lim _{\xi \rightarrow \pm \infty} \frac{d}{d \xi} \Psi(\xi)=0,
$$

Together, boundary conditions (13a)-(13e) supplement quadrature equation (12) and allow one to find the desired grey soliton by direct integration.

\section{Exact analytical grey solitons}

Since the right-hand side of Eq. (12) is an expression cubic in $\rho$, one may write $(d \rho / d \xi)^{2}=B\left(\rho_{0}-\rho\right)^{2}(\rho-$ $\rho_{1}$ ), where $B$ is a constant, $\rho_{0}$ is a double root, and $\rho_{1}$ is a single root. Comparing the coefficients of the four powers of $\rho$ (from 3 to 0 ) uncovers the following relations, respectively: $B=4$,

$$
\left[(2 \kappa K)^{2}+2 \kappa \rho_{0} F^{2}\right] V_{0}^{2}-2 \alpha(2 \kappa K) V_{0}+\left(\alpha^{2}-\rho_{0} F^{2}\right)=0,
$$

$c_{2}=4 \rho_{0}^{2}\left(3-2 A^{2}\right)$, and $c_{1}^{2}=\rho_{0}^{3} F^{2}$, respectively Here the notation $A^{2}+F^{2}=1$, where $F^{2} \equiv \rho_{1} / \rho_{0}$ is the traditional grey soliton contrast parameter, has been introduced. Since $d \rho / d \xi>0$ in the domain $\xi>0$, direct integration of $d \rho / d \xi=2\left(\rho_{0}-\rho\right)\left(\rho-\rho_{1}\right)^{1 / 2}$ gives rise to the classic dark-soliton intensity distribution for a cubic 
nonlinearity, namely $\rho(\xi)=\rho_{0}\left[1-A^{2} \operatorname{sech}^{2}\left(\rho_{0}{ }^{1 / 2} A \xi\right)\right]$. One must also consider the phase distribution. Since the phase gradient $d \Psi / d \xi$ vanishes as $\xi \rightarrow \pm \infty$, it follows that $\left(\alpha-2 \kappa K V_{0}\right)\left(1-2 \kappa V_{0}^{2}\right)^{-1 / 2}+\rho_{0}{ }^{1 / 2} F=0$ [we also note that this result is formally identical to Eq. (14)]. Substituting for $\rho(\xi)$, separation and integration of the phase quadrature equation $d \Psi / d \xi=\rho_{0}^{1 / 2} F\left\{\left[1-A^{2} \operatorname{sech}^{2}\left(\rho_{0}^{1 / 2} A \xi\right)\right]^{-1}-1\right\}$ leads to a second classic result, namely $\Psi(\xi)=\tan ^{-1}\left[(A / F) \tanh \left(\rho_{0}^{1 / 2} A \xi\right)\right]$.

By reintroducing the original space-time coordinates $\tau$ and $\zeta$, the exact analytical dark solitons of Eq. (1) may be written as

$$
\begin{aligned}
u(\tau, \zeta) & =\rho_{0}^{1 / 2}\left[1-A^{2} \operatorname{sech}^{2}\left(\rho_{0}^{1 / 2} A \frac{\tau \mp V_{0} \zeta}{\sqrt{1-2 \kappa V_{0}^{2}}}\right)\right]^{1 / 2} \\
& \times \exp \left\{i \tan ^{-1}\left[\left(\frac{A}{F}\right) \tanh \left(\rho_{0}^{1 / 2} A \frac{\tau \mp V_{0} \zeta}{\sqrt{1-2 \kappa V_{0}^{2}}}\right)\right]\right\} \\
& \times \exp \left[ \pm i \sqrt{1+4 \kappa \beta} \frac{\zeta}{2 \kappa}\right] \exp \left(-i \frac{\zeta}{2 \kappa}\right) .
\end{aligned}
$$

Here, the upper (lower) signs describe a dark soliton evolving forwards in time, and travelling in the forward (backward) longitudinal direction (see Fig. 4). The intrinsic velocity $V_{0}$ is given by

$$
V_{0}(F)=\frac{\rho_{0}^{1 / 2} F \sqrt{1+2 \kappa \beta\left(2+F^{2}\right)-2 \kappa \alpha^{2}}+\alpha \sqrt{1+4 \kappa \beta}}{1+2 \kappa \beta\left(2+F^{2}\right)} .
$$

This generalized expression is obtained by solving Eq. (14), which is quadratic in $V_{0}$ and thus possesses two solutions. However, for compactness of notation we have absorbed these two possible sign choices into the argument of the tanh and sech functions [c.f. solution (15)], and hence $V_{0}$ must be always non-negative. The above expressions yield consistency with those found for black solitons (whose results are flagged by the ' $b$ ' subscript), $F=0$ and $V_{0}(0) \equiv V_{0 b}$, where (see Fig. 5)

$$
V_{0 \mathrm{~b}} \equiv \frac{\alpha}{\sqrt{1+4 \kappa \beta}} \text {. }
$$

The equation for $V_{0 \mathrm{~b}}$ is also identical to that given in Refs. [4,5] for the bright soliton of Eq. (1). It is interesting to note that $V_{0}(F)$ is not anti-symmetric with respect to $F$ since $V_{0}(-F) \neq-V_{0}(F)$ [7,23]. For convenience, one may express solution (15) in the following more compact form:

$$
\begin{aligned}
u(\tau, \zeta) & =\rho_{0}^{1 / 2}\left[A \tanh \left(\rho_{0}^{1 / 2} A \frac{\tau \mp V_{0} \zeta}{\sqrt{1-2 \kappa V_{0}^{2}}}\right)+i F\right] \\
& \times \exp \left[ \pm i \sqrt{1+4 \kappa \beta} \frac{\zeta}{2 \kappa}\right] \exp \left(-i \frac{\zeta}{2 \kappa}\right),
\end{aligned}
$$

where phase variations have been absorbed into the complex tanh-shaped wave profile.

\section{Mapping onto continuous waves}

In the spirit of the frames-of-reference approach [4,5], space-time (geometrical) operations can now be used to find a more general dark soliton of Eq. (1). Such a solution comprises a grey dip modulating a cw 
background field that has a non-zero frequency shift [c.f. Eq. (3b)]. Note that while transformation laws (8a)-(8d) are expressed in terms of velocity $V$, the free parameter should ideally be $\Omega$. One can proceed by establishing the relationship connecting $\Omega$ and $V$. This $\Omega(V)$ correspondence can be uncovered by a twostage analysis: (i) apply geometrical transformation (8) to solution (17); and (ii) map the transformed $\mathrm{cw}$ component (in terms of $V$ ) onto the solution given in Eq. (3b) (in terms of $\Omega$ ). The first part of this procedure leads to

$$
\begin{aligned}
u(\tau, \zeta) & =\rho_{0}^{1 / 2}\left[A \tanh \left(\rho_{0}^{1 / 2} A \frac{\tau \mp W \zeta}{\sqrt{1-2 \kappa W^{2}}}\right)+i F\right] \\
& \times \exp \left[-i \Omega \tau \pm i \sqrt{1+4 \kappa \beta+4 \kappa \Omega\left(\alpha+\frac{1}{2} \Omega\right)} \frac{\zeta}{2 \kappa}\right] \\
& \times \exp \left(-i \frac{\zeta}{2 \kappa}\right),
\end{aligned}
$$

while the second part (equating the temporal phase slope) yields

$$
\Omega(V) \equiv V \sqrt{\frac{1+4 \kappa \beta}{1-2 \kappa V^{2}}}+\alpha\left(\frac{1}{\sqrt{1-2 \kappa V^{2}}}-1\right) .
$$

With this definition of $\Omega(V)$, the longitudinal phase slope is automatically mapped so that solution (18) is entirely self-consistent. Inversion of Eq. (19a) can also determine $V$ as a function of $\Omega$ :

$$
V(\Omega)=\frac{(\Omega+\alpha) \sqrt{1+4 \kappa \beta+4 \kappa \Omega\left(\alpha+\frac{1}{2} \Omega\right)}-\alpha \sqrt{1+4 \kappa \beta}}{1+4 \kappa \beta+2 \kappa(\Omega+\alpha)^{2}},
$$

where $V(\Omega)$ is independent of $F$ (as must be the case, since soliton greyness is unaffected by any frequency shift in the cw background field). The net velocity $W \equiv W(F, \Omega)$ is given in Eq. (8d), where $V_{0} \equiv V_{0}(F)$ and $V \equiv V(\Omega)$ are obtained from Eqs. (16a) and (19b), respectively. This results in an algebraic expression for $W(F, \Omega)$ that is somewhat cumbersome, namely

$$
W(F, \Omega)=\frac{[(\Omega+\alpha) \sqrt{\eta}-\alpha \sqrt{1+4 \kappa \beta}] \mu+\left[\rho_{0}^{1 / 2} F \sqrt{\mu-2 \kappa \alpha^{2}}+\alpha \sqrt{1+4 \kappa \beta}\right]\left(\eta+2 \kappa \alpha^{2}\right)}{\left(\eta+2 \kappa \alpha^{2}\right) \mu+2 \kappa[(\Omega+\alpha) \sqrt{\eta}-\alpha \sqrt{1+4 \kappa \beta}]\left[\rho_{0}^{1 / 2} F \sqrt{\mu-2 \kappa \alpha^{2}}+\alpha \sqrt{1+4 \kappa \beta}\right]}
$$

where, for compactness, we have introduced $\eta \equiv 1+4 \kappa \beta+4 \kappa \Omega(\alpha+\Omega / 2)$ and $\mu \equiv 1+2 \kappa \beta\left(2+F^{2}\right)$. For black solitons, Eq. (20a) simplifies considerably to $W(0, \Omega) \equiv W_{\mathrm{b}}(\Omega)$, which is given by

$$
W_{\mathrm{b}}(\Omega)=\frac{\alpha+\Omega}{\sqrt{1+4 \kappa \beta+4 \kappa \Omega\left(\alpha+\frac{1}{2} \Omega\right)}} .
$$

Equation (20b) agrees with the result in Refs. [4,5], which was derived for bright and black solitons on a slightly different basis (though still with frame-of-reference ideas in mind). One may also prove that $W_{\mathrm{b}}$ is compatible with transformation laws (8a)-(8d), since it can be shown that $W_{\mathrm{b}}(\Omega)=\left[V_{0 \mathrm{~b}}+V(\Omega)\right] /[1+$ $\left.2 \kappa V_{0 \mathrm{~b}} V(\Omega)\right]$. It is interesting to note that an ansatz approach fails to yield solution (18). 


\section{E. Exact analytical snoidal waves}

Model (1) supports classes of nonlinear waves that are periodic in $\tau$ [26]. While a soliton is typically a single localized entity, temporally extended solutions (e.g., described by Jacobi elliptic functions of the first kind) represent periodic wavetrains of similar pulses whose separation (in time) is controlled by the modulus $m$, where $0 \leq m \leq 1$. In the limit $m \rightarrow 1$, the period becomes infinite and the solution reduces to a single soliton pulse. The analytical form of such periodic waves was recently reported in Ref. [5] for the anomalous-GVD regime $(s=+1)$, where families of cnoidal and dnoidal families were uncovered. Families of snoidal wave, that exist in the normal-GVD regime $(s=-1)$, are reported here:

$$
\begin{aligned}
u(\tau, \zeta) & =m \rho_{0}^{1 / 2} \operatorname{sn}\left(\rho_{0}^{1 / 2} \frac{\tau \mp W_{\mathrm{sn}} \zeta}{\sqrt{1-2 \kappa W_{\mathrm{sn}}^{2}}} ; m\right) \\
& \times \exp \left[-i \Omega \tau \pm i \sqrt{1+4 \kappa \beta_{\mathrm{sn}}+4 \kappa \Omega\left(\alpha+\frac{1}{2} \Omega\right)} \frac{\zeta}{2 \kappa}\right] \\
& \times \exp \left(-i \frac{\zeta}{2 \kappa}\right)
\end{aligned}
$$

where $\beta_{\text {sn }} \equiv \rho_{0}\left(1+m^{2}\right) / 2$. The net velocity $W_{\text {sn }}$ of the $s n$ wave is formally identical to $W_{\mathrm{b}}$ [see Eq. (20b)], except that the propagation constant $\beta$ is replaced by $\beta_{\mathrm{sn}}$. For $m \rightarrow 1$, the $s n$ function becomes a tanh and, in this case, a black soliton emerges. As $m \rightarrow 0$, solution (21) tends to a (quasi-linear) sin wave with vanishingly-small amplitude.

\section{F. Velocity representation}

Thus far, exact analytical dark solitons of Eq. (1) have been presented using a notation that corresponds to that traditionally encountered in pulse physics (i.e., where the temporal phase gradient, identified with a frequency shift, is treated as a free parameter). It is also possible to represent the same solutions using a velocity-type representation of the phase (one that is more frequently used when describing nonlinear beams). In a sense, this is a more natural representation, given the velocity parameterization of transformation laws (8a)-(8d). Dark soliton (18) then becomes

$$
\begin{aligned}
u(\tau, \zeta) & =\rho_{0}^{1 / 2}\left[A \tanh \left(\rho_{0}^{1 / 2} A \frac{\tau \mp W \zeta}{\sqrt{1-2 \kappa W^{2}}}\right)+i F\right] \\
& \times \exp \left[i \sqrt{\frac{1+4 \kappa \beta}{1-2 \kappa V^{2}}}\left(-V \tau \pm \frac{\zeta}{2 \kappa}\right)\right] \\
& \times \exp \left(-i \alpha \frac{\tau \mp V \zeta}{\sqrt{1-2 \kappa V^{2}}}+i \alpha \tau\right) \exp \left(-i \frac{\zeta}{2 \kappa}\right),
\end{aligned}
$$

where $V$ is the free parameter, while $V_{0}$ and $W$ are given by Eqs. (16a) and (8d), respectively. In a similar way, one can express snoidal wave (21) in the form 


$$
\begin{aligned}
u(\tau, \zeta) & =m \rho_{0}^{1 / 2} \operatorname{sn}\left(\rho_{0}^{1 / 2} \frac{\tau \mp W_{\mathrm{sn}} \zeta}{\sqrt{1-2 \kappa W_{\mathrm{sn}}^{2}}} ; m\right) \\
& \times \exp \left[i \sqrt{\frac{1+4 \kappa \beta_{\mathrm{sn}}}{1-2 \kappa V^{2}}}\left(-V \tau \pm \frac{\zeta}{2 \kappa}\right)\right] \\
& \times \exp \left(-i \alpha \frac{\tau \mp V \zeta}{\sqrt{1-2 \kappa V^{2}}}+i \alpha \tau\right) \exp \left(-i \frac{\zeta}{2 \kappa}\right)
\end{aligned}
$$

where $W_{\mathrm{sn}}=\left(V_{0 \mathrm{sn}}+V\right) /\left(1+2 \kappa V V_{0 \mathrm{sn}}\right), V$ is the free parameter and $V_{0 \mathrm{sn}}$ is identical to $V_{0 \mathrm{~b}}$ [see Eq. (16b)]. This representation is advantageous for drawing comparisons with the mathematical structure of spatial solitons, and seeing more clearly the effect on exact analytical solutions of including the term $i \alpha \partial u / \partial \tau$ in the governing equation. Note that, when deriving the backward solutions in Eqs. (22a) and (22b), it was convenient to make the change $V \rightarrow-V$ in Eqs. (8a)-(8d). This swap merely preserves the position of the + and - signs in Eq. (8d), and makes for a more compact simultaneous representation of forward and backward pulses.

\section{CONVENTIONAL DARK SOLITONS \\ AND SNOIDAL WAVES}

It is axiomatic that, when all contributions from $\kappa \partial^{2} u / \partial \zeta^{2}$ are negligible simultaneously, the corresponding predictions of conventional pulse theory [i.e., Eq. (2)] must emerge from Eq. (1). However, this physically intuitive requirement is more subtle to implement mathematically than simply setting $\kappa=0$. Indeed, experience has shown that this over-simplified approach yields erroneous results in the spatial domain [25], and this is also true here. In the reduction to conventional pulse theory, one must pay careful attention to the way $\kappa$ interplays with other parameters. As illustrated in Sec. III.B, the formal restriction $\kappa \partial^{2} u / \partial \zeta^{2} \rightarrow 0$ when applied to solutions of Eq. (1) is fully equivalent to a multi-fold algebraic limit.

It is particularly instructive to consider transformation laws (8a)-(8c). Under the restriction $\kappa V^{2} \rightarrow 0$, one recovers $\tau \simeq \tau^{\prime}-V \zeta^{\prime}, \quad \zeta \simeq \zeta^{\prime}$, and $u(\tau, \zeta) \simeq \exp \left[-i s V \tau^{\prime}+i s\left(V^{2} / 2+\alpha V\right) \zeta^{\prime}\right] u^{\prime}\left(\tau^{\prime}, \zeta^{\prime}\right)$. In the limit $\kappa V_{0} V \rightarrow 0$, the familiar Galilean (additive) velocity combination rule is recovered from Eq. (8d), namely $W$ $\simeq V_{0}+V$.

\section{A. Dark solitons}

The multiple limit one has to enforce in solution (18) is $\kappa \rightarrow 0$ (negligible spatial dispersion), $\kappa \beta \rightarrow 0$ (negligible nonlinear phase shift), and $\kappa \Omega(\alpha+\Omega / 2) \rightarrow 0$ (negligible frequency shift). Applying this limit to the intrinsic and background velocities, $V_{0}$ [see Eq. (16b)] and $V$ [see Eq. (19b)], respectively, one finds that to leading order: $V_{0} \simeq \rho_{0}{ }^{1 / 2} F+\alpha$ and $V \simeq \Omega$. This latter result illustrates the important point that frequency shifts and velocities are completely interchangeable in conventional theory: they have the same mathematical status under the SVEA, though Eqs. (16b) and (19b) show that the same is clearly not true in the more general framework. The net velocity $W$ of the grey soliton becomes $W \simeq \rho_{0}{ }^{1 / 2} F+\alpha+\Omega \simeq V_{0}+V$ [see Eqs. 
(20a) and (8d)], which is the familiar Galilean velocity combination rule. Solution (18) may then be expressed as

$$
\begin{aligned}
& u(\tau, \zeta) \simeq \rho_{0}^{1 / 2}\left\{A \tanh \left[\rho_{0}^{1 / 2} A \Theta(\tau, \zeta)\right]+i F\right\} \\
& \times \exp \left[-i \Omega(\tau \mp \alpha \zeta) \pm i\left(\beta+\frac{\Omega^{2}}{2}\right) \zeta\right] \\
& \times \exp \left[-i(1 \mp 1) \frac{\zeta}{2 \kappa}\right]
\end{aligned}
$$

where

$$
\Theta(\tau, \zeta) \equiv(\tau \mp \alpha \zeta) \mp\left(\rho_{0}^{1 / 2} F+\Omega\right) \zeta .
$$

Direct substitution verifies that, on the one hand, the approximate forward solution satisfies Eq. (2) exactly. On the other hand, the backward solution does not satisfy that equation because the rapid phase term, namely $\exp [-i 2(\zeta / 2 \kappa)]$, remains. This situation points to the intrinsic uni-directionality of wave equations derived on the assumption of slowly-varying envelopes. In the local time frame $\left(\tau_{\mathrm{loc}}, \zeta_{\mathrm{loc}}\right)=(\tau-\alpha \zeta, \zeta)$, the forward solution transforms into the classic form [7]

$$
\begin{aligned}
u\left(\tau_{\mathrm{loc}}, \zeta_{\mathrm{loc}}\right) & \simeq \rho_{0}^{1 / 2}\left\{A \tanh \left[\rho_{0}^{1 / 2} A\left(\tau_{\mathrm{loc}}-W_{\mathrm{loc}} \zeta_{\mathrm{loc}}\right)\right]+i F\right\} \\
& \times \exp \left[-i \Omega \tau_{\mathrm{loc}}+i\left(\beta+\frac{\Omega^{2}}{2}\right) \zeta_{\mathrm{loc}}\right]
\end{aligned}
$$

whose net velocity is simply $W_{\text {loc }} \equiv \rho_{0}{ }^{1 / 2} F+\Omega$. The product $\rho_{0}{ }^{1 / 2} F$ thus plays the role of a local intrinsic velocity, so that when $\Omega=0$ (zero frequency shift) and $F=0$ (black soliton), the pulse is stationary in the local time frame (i.e., the intensity minimum moves along the line $\zeta_{\text {loc }}=0$ because one has $W_{\text {loc }}=0$ ).

\section{B. Interpretation of intrinsic velocity}

To gain deeper insight into soliton velocities, it is instructive to consider coordinate transformations more carefully. Without loss of generality, we restrict our attention to the $\Omega=0$ subset of solutions (we note that inclusion of a finite frequency shift affects none of the following).

A physical distinction between black (i.e., $F=0$ ) and grey (i.e., $|F|>0$ ) dark solitons is the size of the phase shift $\Delta \Psi$ across their temporal profile (being $\pi$ radians and, generally, less than $\pi$ radians, respectively). In conventional pulse theory, where one tends to analyze waves in local coordinates ( $\left.\tau_{\text {loc }}, \zeta_{\text {loc }}\right)$, this phase shift, namely $\Delta \Psi=-2 \tan ^{-1}(A / F)=2 \tan ^{-1}(F / A)-\pi$ [2], is manifest as a so-called intrinsic velocity $\rho_{0}{ }^{1 / 2} F$ [c.f. solution (24)]. By invoking the inverse Galilean transformation, the same dark soliton when expressed in $(\tau, \zeta)$ coordinates [c.f. solution (23) with the upper signs] is governed by Eq. (2) and its intrinsic velocity becomes $\rho_{0}^{1 / 2} F+\alpha$. Hence, from the frames-of-reference perspective, both grey and black solitons (and, for that matter, bright solitons also [4,5]) must have an intrinsic velocity with respect to the laboratory frame (by virtue of simply travelling along the $z$ axis). Note that $\Delta \Psi$ is preserved (as it must be) when transforming between local and laboratory frames. 
The way in which velocities combine under Galilean-type boosts oversimplifies these considerations. The separation of intrinsic velocity into two additive factors is merely an artefact of the SVEA; furthermore, one of these factors may be transformed away through a change in coordinates. Equation (16a) shows that in the more general case, $V_{0}(F)$ has a much stronger geometrical characteristic than has previously been recognized. The interplay between system and solution parameters is then more intricate, and the intrinsic velocity cannot be broken up in such a straightforward and intuitive way.

\section{Snoidal waves}

Applying a similar asymptotic procedure, snoidal wave (21) is approximated by

$$
\begin{aligned}
u(\tau, \zeta) \simeq & m \rho_{0}^{1 / 2} \operatorname{sn}\left[\rho_{0}^{1 / 2} \Theta_{\mathrm{sn}}(\tau, \zeta) ; m\right] \\
& \times \exp \left[-i \Omega(\tau \mp \alpha \zeta) \pm i\left(\beta_{\mathrm{sn}}+\frac{\Omega^{2}}{2}\right) \zeta\right] \\
& \times \exp \left[-i(1 \mp 1) \frac{\zeta}{2 \kappa}\right],
\end{aligned}
$$

where $\Theta_{\mathrm{sn}}(\tau, \zeta) \simeq(\tau \mp \alpha \zeta) \mp \Omega \zeta$. Direct substitution verifies that the approximate forward wave satisfies Eq. (2) exactly, while the approximate backward wave does not. In the local time frame $\left(\tau_{\text {loc }}, \zeta_{\text {loc }}\right)=(\tau-\alpha \zeta$, $\zeta)$, the forward solution transforms into the classic form [26]

$$
\begin{aligned}
u\left(\tau_{\mathrm{loc}}, \zeta_{\mathrm{loc}}\right) & \simeq m \rho_{0}^{1 / 2} \operatorname{sn}\left[\rho_{0}^{1 / 2}\left(\tau_{\mathrm{loc}}-\Omega \zeta_{\mathrm{loc}}\right) ; m\right] \\
& \times \exp \left[-i \Omega \tau_{\mathrm{loc}}+i\left(\beta_{\mathrm{sn}}+\frac{\Omega^{2}}{2}\right) \zeta_{\mathrm{loc}}\right],
\end{aligned}
$$

where the net velocity in this frame is just the frequency shift $\Omega$.

\section{STABILITY OF DARK SOLITON PULSES}

\section{A. Stability criterion}

Conventional nonlinear theories of dark soliton stability are routinely performed in the local time frame. They are also based on a renormalization procedure, whereby the continuous infinity of degrees-of-freedom associated with the cw background is subtracted in a self-consistent way, rendering the system's conserved quantities (energy-flow, momentum, and Hamiltonian) finite [2,27]. To facilitate analysis, one usually considers the $\Omega=0$ subset of solutions. The stability criterion is introduced by way of a renormalized momentum integral $M_{\text {ren }}$ [28]. A dark solution is then predicted to be stable against small perturbations if $d M_{\text {ren }} / d V_{0}$ $>0$, where

$$
M_{\mathrm{ren}}\left(V_{0}\right) \equiv \frac{i}{2} \int_{-\infty}^{+\infty} d \tau_{\mathrm{loc}}\left(u^{*} \frac{\partial u}{\partial \tau_{\mathrm{loc}}}-u \frac{\partial u^{*}}{\partial \tau_{\mathrm{loc}}}\right)\left(1-\frac{\rho_{0}}{|u|^{2}}\right),
$$

$\rho_{0}$ is the intensity of the cw background, and $V_{0} \equiv \rho_{0}{ }^{1 / 2} F$ is the intrinsic velocity of the dark component in the local time frame. Substituting solution (24) into Eq. (27), it can be shown that $M_{\text {ren }}\left(V_{0}\right)=2 V_{0}\left(\rho_{0}-V_{0}^{2}\right)^{1 / 2}-$ $2 \rho_{0} \tan ^{-1}\left[\left(\rho_{0}-V_{0}^{2}\right)^{1 / 2} / V_{0}\right]$. 
In the more general model (1), one might expect a relationship similar to Eq. (27) to hold on physical grounds. If a pulse (either bright or dark) is predicted to be stable in its local time frame, then for that prediction to be meaningful, the same pulse must also be stable when viewed from any other frame (e.g., the laboratory). Symmetry principles mean that one does not expect instabilities to appear and disappear spontaneously when boosting between different coordinate systems (since the choice of reference frame is an entirely arbitrary one). The formal mathematical question of stability criteria for bright and dark (spatial and temporal) Helmholtz solitons remains open, as does the issue of renormalization theory for classes of fullysecond order nonlinear envelope equations such as model (1). However, one can still address questions conerning dark soliton stability through initial value problems and numerical computation.

\section{B. Initial value problems}

Simulations are now used to probe the fully-developed nonlinear dynamics when Helmholtz dark pulses suffer a perturbation to their temporal shape. We begin our investigation by injecting a perturbed black $(F=$ 0) solution,

$$
u(\tau, 0)=\rho_{0}^{1 / 2} \tanh \left(\rho_{0}^{1 / 2} \tau\right) \exp (-i \Omega \tau),
$$

of full width $\Delta \tau_{0} \equiv 2 / \rho_{0}^{1 / 2}$, into the system. The formal shape perturbation arises from omission of the correction factor $\left(1-2 \kappa W^{2}\right)^{1 / 2}$ from the envelope. Since this input pulse corresponds to an exact solution of Eq. (2) [c.f. soliton (23a) with upper signs], this class of initial value problem considers propagation effects when one does not take full account of contributions from the $\kappa \partial^{2} u / \partial \zeta^{2}$ term [23].

Extensive computations with Eq. (1) have been performed using a generalization of the differencedifferential algorithm in Ref. [29] to allow for the $i \alpha \partial u / \partial \tau$ term. As the pulse travels along $\zeta$, its width evolves toward the asymptotic value $\Delta \tau_{\infty}=\left(1-2 \kappa W^{2}\right)^{1 / 2} \Delta \tau_{0}$, corresponding to the theoretical prediction for an exact (stationary) dark soliton [23]. Self-reshaping is accompanied by the emission of a small amount of radiation. Figure 6 presents two illustrative sets of results for typical pulse evolution. When $\kappa>0$ (the hyperbolic scenario), the injected pulse is initially too long for the cw background intensity $\rho_{0}$. The interplay between dispersion and nonlinearity thus tends to shorten the pulse length. Similarly, for $\kappa<0$ (the elliptic scenario) the injected pulse is too short in time. The solution thus tends to broaden as it propagates.

The robustness of grey solutions can be addressed using an initial condition of the form $u(\tau, 0)=$ $\rho_{0}{ }^{1 / 2}\left[A \tanh \left(\rho_{0}{ }^{1 / 2} A \tau\right)+i F\right]$, whose full-width is $\Delta \tau_{0} \equiv 2 / \rho_{0}{ }^{1 / 2} A$. This input pulse corresponds to an exact grey soliton of the conventional model equation with $V(\Omega=0)=0$ and hence $W=V_{0}$ [where $V_{0}$ is given by Eq. (16a)]. The self-reshaping characteristics of perturbed grey solitons are qualitatively similar to those of the black solutions shown in Fig. 6. A key quantitative difference is that much longer propagation lengths are required for stationary grey solitons to emerge from the initial condition. 


\section{CONCLUSIONS}

A framework for describing dark pulses of a universal governing equation with second-order spatiotemporal dispersion and a cubic nonlinearity has been examined. Linear analysis has predicted the MI characteristics of continuous waves, with simulations confirming theoretical predictions. New families of exact analytical dark soliton have been derived for normal-GVD regimes, along with a class of (temporally-periodic) snoidal wave. The space-time geometry of these new solutions has been explored in detail. Furthermore, computations provided supporting evidence that these dark solitons may be classified as robust fixed-point attractors of the system dynamics.

The analyses presented here, and also in Refs. 4 and 5, take the first steps toward a deeper understanding of nonlinear pulses in fully second-order systems. Many other exciting avenues of theoretical and technological importance remain to be explored. Most obviously, perhaps, is the question of spatiotemporal soliton pulse multiplexing [30] (an adiabatic perturbation method for Helmholtz-type beam systems was developed in Ref. 31). Equation (1) can be modified to include other generic nonlinearities (particularly those functional forms where one might now reasonably expect to find exact analytical solitons). Also of interest is the potential for generalizing model (1) to multi-component regimes [32]. Given the detailed knowledge now established for scalar bright [4,5] and dark Helmholtz solitons in the time domain, vector contexts [33] are especially appealing and a natural candidate for further study. Such coupled-mode equations could be used in photonics applications to understand how light behaves in birefringent waveguides [34] in the presence of spatiotemporal dispersion. Moreover, since spatial dispersion appears generically from the linear wave equation Laplacian, one may reasonably expect Helmholtz soliton pulses in a range of other, non-optical, contexts.

\section{REFERENCES}

[1] Y. S. Kivshar and B. A. Malomed, Rev. Mod. Phys. 61, 763 (1989); A. C. Scott, F. Y. F. Chu, and D. W. McLaughlin, Proc. IEEE 61, 1443 (1973).

[2] Y. S. Kivshar and B. Luther-Davies, Phys. Rep. 298, 81 (1998); Y. S. Kivshar, IEEE J. Quantum Electron. 29, 250 (1993).

[3] R. K. Dodd, J. C. Eilbeck, J. D. Gibbon, and H. C. Morris, Solitons and Nonlinear Wave Equations (Academic Press, London 1982); G. L. Lamb, Elements of Soliton Theory (John Wiley \& Sons, New York, 1980).

[4] J. M. Christian et al., Phys. Rev. Lett. 108, 034101 (2012).

[5] J. M. Christian et al., "Wave envelopes with second-order spatiotemporal dispersion: I. Kerr bright solitons and cnoidal waves," submitted to Phys. Rev. A (2012).

[6] R. W. Boyd, Nonlinear Optics 2nd Ed. (Academic Press, San Diego, 2003); K. J. Blow and N. J. Doran, "Solitons in optical fibres," pp. 325 in Nonlinear Waves in Solid State Physics, ed. A. D. Boardman et al. (Plenum Press, New York, 1990); Kh. I. Pushkarov, D. I. Pushkarov, and I. V. Tomov, Opt. Quantum Electon. 11, 471 (1979).

[7] V. E. Zakharov and A. B. Shabat, Sov. Phys. JETP 34, 62 (1972); ibid 37, 823 (1973). 
[8] A. Hasegawa and F. Tappert, Appl. Phys. Lett. 23, 142 (1973); ibid 171 (1973); L. F. Mollenauer, R. H. Stolen, and J. P. Gordon, Phys. Rev. Lett. 45, 1095 (1980); L. F. Mollenauer et al., Opt. Lett. 15, 1203 (1973).

[9] Y. Kodama and A. Hasegawa, IEEE J. Quantum Electron. QE-23, 510 (1987).

[10] S. A. Gredeskul and Y. S. Kivshar, Opt. Lett. 14, 1281 (1989); Y. S. Kivshar, J. Phys. A: Math. Gen. 22, 337 (1989).

[11] N. J. Doran and D. Wood, J. Opt. Soc. Am. B 4, 1843 (1987); K. J. Blow and D. Wood, Opt. Commun. 58, 349 (1986); K. J. Blow and N. J. Doran, Phys. Lett. 107A, 55 (1985).

[12] V. Karpman, Phys. Rev. E 47, 2073 (1993); Y. S. Kivshar, Phys. Rev. A 43, 1677 (1991); Opt. Lett. 16, 892 (1991); P. K. A. Wai et al., Opt. Lett. 11, 464 (1986).

[13] A. Sakovich and S. Sakovich, J. Phys. A: Math. Gen 39, L361 (2006); Y. Chung, C. K. R. T. Jones, T. Schäfter, and C. E. Wayne, Nonlinearity 18, 1351 (2005); T. Schäfer and C. E. Wayne, Physica D 196, 90 (2004).

[14] F. Biancalana and C. Creatore, Opt. Exp. 16, 14882 (2008); V. M. Agranovich and V. L. Ginzburg, Crystal Optics with Spatial Dispersion, and Excitons (Springer, Berlin, 1984).

[15] M. G. Vakhitov and A. A. Kolokolov, Radiophys. Quantum Electron. 16, 783 (1973); J. Satsuma and N. Yajima, Suppl. Prog. Theor. Phys. 55, 284 (1974).

[16] Y. S. Kivshar, D. Anderson, and M. Lisak, Phys. Scr. 47, 679 (1993).

[17] N. Akhmediev, W. Królikowski, and A. J. Lowery, Opt. Commun. 131, 260 (1996); Y. S. Kivshar and V. V. Afansasjev, Opt. Lett. 16, 285 (1991); Y. S. Kivshar, Phys. Rev. A 42, 1757 (1990).

[18] G. Fibich, W. Ren, and X.-P. Wang, Phys. Rev. E 67056603 (2003); G. Fibish and G. C. Papanicolaou, Opt. Lett. 22, 1379 (1997); J. E. Rothenberg, Opt. Lett. 17, 1340 (1992).

[19] J. M. Christian, G. S. McDonald, and P. Chamorro-Posada, J. Phys. A: Math. Theor. 40, 1545 (2007); ibid. Corrigendum 40, 8601 (2007).

[20] M. Abramowitz and I. A. Stegun, Handbook of mathematical functions with formulas, graphs, and mathematical tables (Dover, New York, 1972).

[21] Y. S. Kivshar, D. Anderson, and M. Lisak, Phys. Scr. 47, 679 (1993);

[22] J. M. Christian, G. S. McDonald, and P. Chamorro-Posada, Phys. Rev. A 81, 053831 (2010).

[23] P. Chamorro-Posada and G. S. McDonald, Opt. Lett. 28, 825 (2003).

[24] J. D. Jackson, Classical Electrodynamics 3rd Ed. (John Wiley, New York, 1999); H. Goldstein, Classical Mechanics, 2nd Ed. (Addison-Wesley, Philippines, 1980).

[25] P. Chamorro-Posada, G. S. McDonald, and G. H. C. New, J. Mod. Opt. 47, 1877 (2000); ibid. 45, 1111 (1998).

[26] Y. V. Kartashov et al., Phys. Rev. E 67, 036613 (2003); Y. V. Kartashov et al., J. Opt. Soc. Am. B 30, 1273 (2003); V. Aleshkevich, Y. Kartashov, and V. Vysloukh, Opt. Commun. 185, 305 (2000); V. P. Kudashev and A. B. Mikhă̌lovskiı̌, Sov. Phys. JETP 63, 972 (1986).

[27] Y. S. Kivshar and X. Yang, Phys. Rev. E 49, 1657 (1994). 
[28] D. E. Pelinovsky, Y. S. Kivshar, and V. V. Afanasjev, Phys. Rev. E 54, 2015 (1996); I. V. Barashenkov, Phys. Rev. Lett. 77, 1193 (1996).

[29] P. Chamorro-Posada, G. S. McDonald, and G. H. C. New, Opt. Commun. 192, 1 (2001).

[30] M. J. Ablowitz et al., J. Opt. Soc. Am. B 14, 1788 (1997); J. P. Gordon, Opt. Lett. 8, 596 (1983).

[31] P. Chamorro-Posada and G. S. McDonald, Phys. Rev. E 74, 036609 (2006).

[32] S. V. Manakov, Sov. Phys. JETP 38, 248 (1974); J. M. Christian, G. S. McDonald, and P. ChamorroPosada, Phys. Rev. E 74, 066612 (2006).

[33] Y. S. Kivshar and S. K. Turitsyn, Opt. Lett. 18, 227 (1993); V. K. Mesentsev and S. K. Turitsyn, ibid 17, 1497 (1992); Y. S. Kivshar, ibid 17, 1322 (1992); V. V Afanasjev et al., ibid 14, 805 (1989); D. N. Christodoulides and R. I. Joseph, ibid 13, 53 (1988).

[34] C. R. Menyuk, J. Opt. Soc. Am. B 5, 392 (1988); Opt. Lett. 12, 614 (1987); IEEE J. Quantum Electron. 23, 174 (1987).

\section{APPENDIX}

\section{A. Derivation of optical model equation}

In the classic scalar wave optics approach, the transverse spatial profile of the electric field $\mathbf{E}$ is confined by the structure of a waveguide and the polarization scrambling term in Maxwell's equations, namely $\nabla(\nabla \cdot \mathbf{E})$, can be safely neglected. One proceeds by seeking pulse solutions that have the form $E(t, z)=$ $A(t, z) \exp \left[i\left(k_{0} z-\omega_{0} t\right)\right]+A^{*}(t, z) \exp \left[-i\left(k_{0} z-\omega_{0} t\right)\right]$, where $z$ is the longitudinal coordinate along the axis of the waveguide, $t$ denotes the time coordinate, and $A(t, z)$ is the envelope. The underlying carrier wave has a centre frequency $\omega_{0}$ and propagation constant $k_{0}=n_{0} \omega_{0} / c$, where $n_{0}$ is the linear refractive index of the host medium (at frequency $\omega_{0}$ ) and $c$ is the speed of light in a vacuum.

By substituting the field $E$ into the corresponding Maxwell equations and transforming to the temporal frequency domain (denoted by $\omega$ ), it can be shown that [6]

$$
\frac{\partial^{2} \tilde{A}}{\partial z^{2}}+i 2 k_{0} \frac{\partial \tilde{A}}{\partial z}+\left[k^{2}(\omega)-k_{0}^{2}\right] \tilde{A}=0
$$

where $\tilde{A} \equiv \tilde{A}\left(\omega-\omega_{0}, z\right)$ is the Fourier transform of the pulse envelope and $k^{2}$ is the mode eigenvalue (obtained by solving Maxwell equations for the transverse part of the confined field).

At this point, it is customary to deploy a Taylor expansion to deal with the linear temporal dispersive properties of the system that are contained implicitly within $k^{2}(\omega)$. By assuming that pulse solutions have only a narrow spectral width (temporal variations in $A$ are on much longer time-scales than the characteristic period $\sim 1 / \omega_{0}$ ) and that $\tilde{A}$ remains peaked within the vicinity of $\omega_{0}$ (small frequency shifts) one may write

$$
k^{2}\left(\omega-\omega_{0}\right)=\left.\sum_{j=0}^{\infty} \frac{\partial^{j}}{\partial \omega^{j}} k^{2}(\omega)\right|_{\omega=\omega_{0}} \frac{1}{j !}\left(\omega-\omega_{0}\right)^{j} .
$$


By discarding terms beyond the second order in $\left(\omega-\omega_{0}\right)$, where third- and higher-order linear dispersion effects may be neglected [12], it follows that

$$
k^{2}\left(\omega-\omega_{0}\right)=k_{0}^{2}+2 k_{0} k_{1}\left(\omega-\omega_{0}\right)+\left(k_{0} k_{2}+k_{1}^{2}\right)\left(\omega-\omega_{0}\right)^{2},
$$

where $k_{0} \equiv k\left(\omega_{0}\right)$ and $k_{j} \equiv\left(\partial^{j} k / \partial \omega^{j}\right)_{\omega=\omega_{0}}$ for $j=1,2,3, \ldots$ By noting the correspondence $\left(\omega-\omega_{0}\right)^{j}=(i \partial / \partial t)^{j}$, inverse Fourier transformation back to the time domain leads to the following governing equation:

$$
\begin{aligned}
\frac{1}{2 k_{0}} \frac{\partial^{2} A}{\partial z^{2}} & +i\left(\frac{\partial A}{\partial z}+k_{1} \frac{\partial A}{\partial t}\right) \\
& -\frac{k_{2}}{2}\left(1+\frac{k_{1}^{2}}{k_{0} k_{2}}\right) \frac{\partial^{2} A}{\partial t^{2}}+\frac{\omega_{0}}{c} n_{2}|A|^{2} A=0,
\end{aligned}
$$

where $n_{2}$ is the Kerr coefficient (assumed to be frequency independent) and the system nonlinearity has been included heuristically.

In our analysis, we have followed a more traditional approach that simplifies the scalings without loss of generality. Under similar assumptions as before, the term $k^{2}(\omega)-k_{0}{ }^{2}$ is well approximated by $2 k_{0}\left[k(\omega)-k_{0}\right]$, and it is now $k(\omega)$ [rather than $k^{2}(\omega)$ ] that is Taylor-expanded:

$$
k\left(\omega-\omega_{0}\right)=\left.\sum_{j=0}^{\infty} \frac{\partial^{j}}{\partial \omega^{j}} k(\omega)\right|_{\omega=\omega_{0}} \frac{1}{j !}\left(\omega-\omega_{0}\right)^{j} .
$$

Keeping terms up to second order in $\left(\omega-\omega_{0}\right)$ leads to the familiar quadratic approximation

$$
k\left(\omega-\omega_{0}\right)=k_{0}+k_{1}\left(\omega-\omega_{0}\right)+\frac{k_{2}}{2}\left(\omega-\omega_{0}\right)^{2} .
$$

It is interesting to note that there is full agreement between Eqs. (A6) and (A3), up to terms in $\left(\omega-\omega_{0}\right)^{2}$. By inverse Fourier transforming and introducing the nonlinearity in a similar way, one arrives at the classic optical pulse equation,

$$
\frac{1}{2 k_{0}} \frac{\partial^{2} A}{\partial z^{2}}+i\left(\frac{\partial A}{\partial z}+k_{1} \frac{\partial A}{\partial t}\right)-\frac{k_{2}}{2} \frac{\partial^{2} A}{\partial t^{2}}+\frac{\omega_{0}}{c} n_{2}|A|^{2} A=0 .
$$

While Eq. (A4) is (potentially) a more accurate model, we shall instead continue with Eq. (A7) as this form is more commonly encountered in the literature [1-34].

Equations (A7) and (A4) reveal that electromagnetic modes have an intrinsic propagation contribution to $\partial^{2} / \partial z^{2}$ in the form of the $1 / 2 k_{0}$ travelling-wave pre-factor. Biancalana and Creatore [14] have recently shown that light in some semiconductor waveguides (such as $\mathrm{ZnCdSe} / \mathrm{ZnSe}$ superlattices) can also exhibit a potentially dominant material contribution, whose physical origin lies in the coupling of the confined electric field to diffusing excitons. Spatial dispersion appears through a modification to the coefficient of $\partial^{2} A / \partial z^{2}$ in Eqs. (A7), whereby $1 / 2 k_{0}$ is augmented by the exciton term:

$$
\frac{1}{2 k_{0}} \rightarrow \frac{1}{2 k_{0}}+\frac{n_{0} \Gamma \Delta \tilde{\omega}_{0}}{2 \delta \omega^{2} c}
$$


Here, $\Gamma \equiv \hbar / 2 M_{x}^{*}, M_{x}^{*}$ is the effective exciton mass, $\tilde{\omega}_{0}$ is a resonant frequency, $\Delta$ is a dimensionless parameter related to the oscillator strength for the coherent exciton-photon interaction, and $\delta \omega$ is a frequency detuning (for a detailed account, the reader is directed to the Appendix in Ref. 14). It is interesting to note that the second term in Eq. (A8) can, in principle, become negative when $M_{x}^{*}<0$.

After a rescaling of Eq. (A7), the dimensionless envelope $u$ is governed by Eq. (1). The normalized space and time coordinates are $\zeta=z / L$ and $\tau=t / t_{p}$, respectively, where $t_{p}$ is the duration of a reference pulse with dispersion length $L=t_{p}{ }^{2}|| k_{2} \mid$. The sign of the group velocity dispersion is flagged by $s= \pm 1=-\operatorname{sgn}\left(k_{2}\right)(+1$ for anomalous; -1 for normal), and $\alpha \equiv k_{1} t_{p} /\left|k_{2}\right|$. The spatial dispersion parameter is $\kappa=\kappa_{0}+D$, where $\kappa_{0} \equiv$ $1 / 2 k_{0} L=c\left|k_{2}\right| / 2 n_{0} \omega_{0} t_{p}^{2}$ and $D \equiv n_{0} \Gamma \Delta \tilde{\omega}_{0} / 2 \delta \omega^{2} c L=\left|k_{2}\right| n_{0} \Gamma \Delta \tilde{\omega}_{0} / 2 \delta \omega^{2} c t_{p}^{2}$. Finally, $u=A / A_{0}$, where the natural unit of electric field amplitude is $A_{0}=\left(n_{0} / n_{2} k_{0} L\right)^{1 / 2}=\left(n_{0}\left|k_{2}\right| / k_{0} n_{2} t_{p}^{2}\right)^{1 / 2}$.

\section{B. Galilean transformation}

Under the conventional Galilean boost, one transforms to a frame of reference moving along the $z$ axis (in the forward direction) at the group velocity $v_{g}=1 / k_{1}$. Hence, when $t_{\mathrm{loc}}=t-k_{1} z$ and $z_{\mathrm{loc}}=z$, it follows that the operators $\partial / \partial t$ and $\partial / \partial z$ transform according to $\partial / \partial t_{\mathrm{loc}}$ and $\partial / \partial z_{\mathrm{loc}}-k_{1} \partial / \partial t_{\mathrm{loc}}$, respectively. In the $\left(t_{\mathrm{loc}}, z_{\mathrm{loc}}\right)$ frame, Eqs. (A7) and (A4) become

$$
\begin{aligned}
\frac{1}{2 k_{0}} \frac{\partial^{2} A}{\partial z_{\mathrm{loc}}^{2}}+i \frac{\partial A}{\partial z_{\mathrm{loc}}} & -\frac{k_{2}}{2}\left(1-\frac{k_{1}^{2}}{k_{0} k_{2}}\right) \frac{\partial^{2} A}{\partial t_{\mathrm{loc}}^{2}} \\
& -\frac{k_{1}}{k_{0}} \frac{\partial^{2} A}{\partial z_{\mathrm{loc}} \partial t_{\mathrm{loc}}}+\frac{\omega_{0}}{c} n_{2}|A|^{2} A=0
\end{aligned}
$$

and

$$
\begin{aligned}
\frac{1}{2 k_{0}} \frac{\partial^{2} A}{\partial z_{\mathrm{loc}}^{2}}+ & i \frac{\partial A}{\partial z_{\mathrm{loc}}}-\frac{k_{2}}{2} \frac{\partial^{2} A}{\partial t_{\mathrm{loc}}^{2}} \\
& -\frac{k_{1}}{k_{0}} \frac{\partial^{2} A}{\partial z_{\mathrm{loc}} \partial t_{\mathrm{loc}}}+\frac{\omega_{0}}{c} n_{2}|A|^{2} A=0,
\end{aligned}
$$

respectively. The origin of the mixed partial derivative term at $\partial^{2} A / \partial z_{\mathrm{loc}} \partial t_{\mathrm{loc}}$ lies in the $\partial^{2} / \partial z^{2}$ operator [18], and the numerical coefficient of this term is $k_{1} / k_{0}$ in both cases. To simplify considerations, the mixedderivative term could be neglected but this lead to a less-exact model. By abandoning the Galilean boost and remaining in the laboratory frame, one avoids the mixed-derivative complications associated with Eqs. (B1) and (B2). 


\section{FIGURE CAPTIONS}

FIG. 1 (Color online) Dispersion relations for cw solutions with $\kappa=+10^{-3}, \rho_{0}=1.0$, and $\alpha=1.0$. (a) Ellipse when $s=+1$ [representative of regimes with $\operatorname{sgn}(s \kappa)=+1$ ], and (b) hyperbole when $s=-1$ [representative of regimes with $\operatorname{sgn}(s \kappa)=-1]$. The families of curves are centred on $(\Omega, K)=(s \alpha, 0)$, so the dominant effect of varying $\alpha$ is to translate the curves along the $\Omega$ axis. Blue (upper) curves: forward wave. Red (lower) curves: backward wave.

FIG. 2. (Color online) Dispersion relation [obtained by solving Eq. (6) numerically] for Fourier mode (5) when $\kappa=+10^{-3}, \rho_{0}=1.0, \alpha=1.0$, and $s=+1$. Note that there are always four branches because of the quartic nature of Eq. (14). (a) The propagation constant of the perturbation (real part of the complex wavenumber $K_{p}$ ) comprises two ellipses. (b) The spatial growth rate of the mode (imaginary part of $K_{p}$ ) has a short-wave MI at high- $\Omega_{p}$. Inset: Bow-tie-type structure of the classic long-wave MI curve (the additional two roots are zero). These small-scale features in $\Lambda_{p}\left(\Omega_{p}\right)$ are not apparent from visual inspection of the larger-scale plot.

FIG. 3. (Color online) Spontaneous development of modulational instability in the anomalous-GVD regime $(s=+1)$ when $\kappa=+10^{-3}, \alpha=1.0$, and $\rho_{0}=1.0$. In this simulation, the level of noise is set to $0.01 \%\left(\varepsilon=10^{-4}\right)$. The zero-frequency spectral component (i.e., the dominant peak in the Fourier transform that is associated with the $\Omega=0 \mathrm{cw}$ background field) has been filtered from the dataset. The first sidebands that start to grow are centred on the most unstable frequency $\Omega_{p 0} \simeq\left(2 \rho_{0}\right)^{1 / 2}$, as predicted by linear analysis. The results are qualitatively unchanged for $\kappa=-10^{-3}$ because the long-wave instability region is independent of $\kappa$.

FIG. 4. (Color online) Schematic diagram illustrating the geometry of solution (17), and in particular the distinction between pulses propagating in the forward (FWD) and backward (BWD) longitudinal directions. In the $(\tau, \zeta)$ plane, the minimum of the grey dip travels along the line $\tau-V_{0} \zeta=0$ and $\tau+V_{0} \zeta=0$, respectively, where the intrinsic velocity $V_{0}$ is given by Eq. (16a). The trajectory makes an angle $\Xi$ relative to the longitudinal axis, where $\tan \Xi=V_{0}$.

FIG. 5. (Color online) Schematic diagram illustrating the distinction between forward-propagating black and grey dark solitons. The black soliton (those solutions with $F=0$ ) has an intrinsic velocity $V_{0 b}$ [given by Eq. (16b)], while its more general grey counterpart (here, a solution with $F>0$ ) has an intrinsic velocity $V_{0}$ [where $\left.V_{0}(F>0)>V_{0 b}\right]$.

FIG. 6. (Color online) Self-reshaping of perturbed black $(F=0)$ pulses (28) toward an exact soliton with (a) $\kappa$ $=+10^{-3}$ (hyperbolic scenario) and (b) $\kappa=-10^{-3}$ (elliptic scenario - note that the qualitative features of these curves are similar to those in Ref. [23] for Helmholtz spatial dark solitons). Solid bars denote theoretical predictions of the asymptotic full-width $\Delta \tau_{\infty}$. Other parameters: $\rho_{0}=1.0$ and $\alpha=1.0$; note that the cw background of each pulse has no long-wave MI since $s=-1$. 


\section{Figure 1}

\section{J. M. Christian et al.}

\section{Physical Review A}
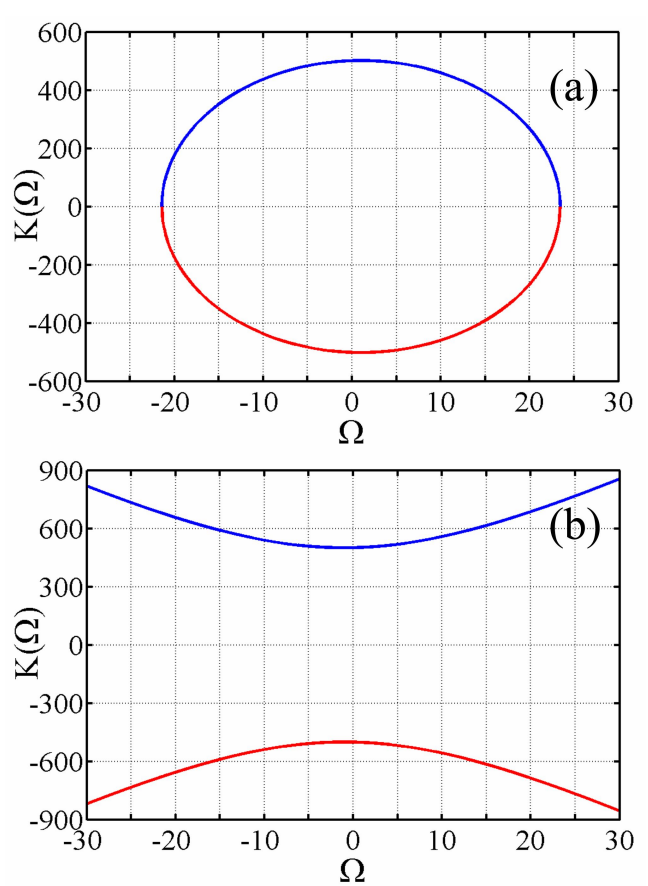

FIG. 1 (Color online) Dispersion relations for cw solutions with $\kappa=+10^{-3}, \rho_{0}=1.0$, and $\alpha=1.0$. (a) Ellipse when $s=+1$ [representative of regimes with $\operatorname{sgn}(s \kappa)=+1$ ], and (b) hyperbole when $s=-1$ [representative of regimes with $\operatorname{sgn}(s \kappa)=-1]$. The families of curves are centred on $(\Omega, K)=(s \alpha, 0)$, so the dominant effect of varying $\alpha$ is to translate the curves along the $\Omega$ axis. Blue (upper) curves: forward wave. Red (lower) curves: backward wave. 


\section{Figure 2}

\section{J. M. Christian et al.}

\section{Physical Review A}
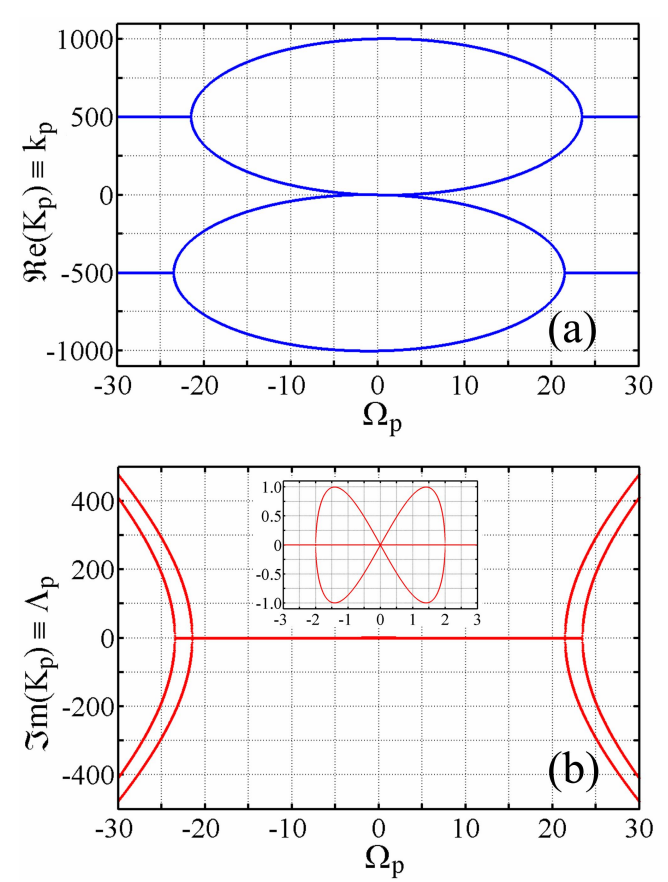

FIG. 2. (Color online) Dispersion relation [obtained by solving Eq. (6) numerically] for Fourier mode (5) when $\kappa=+10^{-3}, \rho_{0}=1.0, \alpha=1.0$, and $s=+1$. Note that there are always four branches because of the quartic nature of Eq. (14). (a) The propagation constant of the perturbation (real part of the complex wavenumber $K_{p}$ ) comprises two ellipses. (b) The spatial growth rate of the mode (imaginary part of $K_{p}$ ) has a short-wave MI at high- $\Omega_{p}$. Inset: Bow-tie-type structure of the classic long-wave MI curve (the additional two roots are zero). These small-scale features in $\Lambda_{p}\left(\Omega_{p}\right)$ are not apparent from visual inspection of the larger-scale plot. 


\section{Figure 3}

\section{J. M. Christian et al.}

\section{Physical Review A}

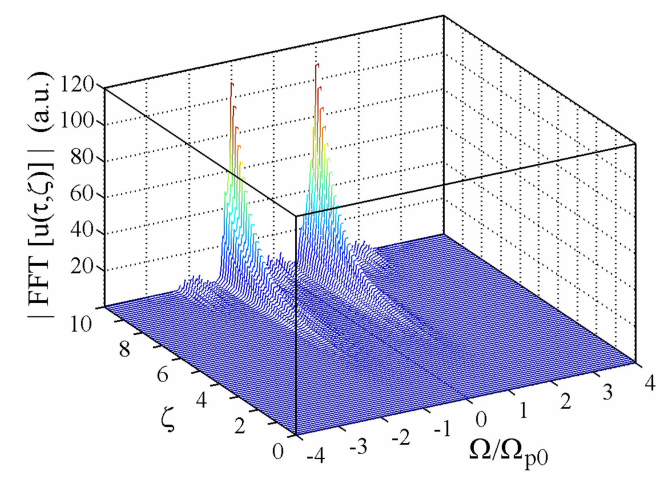

FIG. 3. (Color online) Spontaneous development of modulational instability in the anomalous-GVD regime $(s=+1)$ when $\kappa=+10^{-3}, \alpha=1.0$, and $\rho_{0}=1.0$. In this simulation, the level of noise is set to $0.01 \%\left(\varepsilon=10^{-4}\right)$. The zero-frequency spectral component (i.e., the dominant peak in the Fourier transform that is associated with the $\Omega=0 \mathrm{cw}$ background field) has been filtered from the dataset. The first sidebands that start to grow are centred on the most unstable frequency $\Omega_{p 0} \simeq\left(2 \rho_{0}\right)^{1 / 2}$, as predicted by linear analysis. The results are qualitatively unchanged for $\kappa=-10^{-3}$ because the long-wave instability region is independent of $\kappa$. 


\section{Figure 4}

\section{J. M. Christian et al.}

\section{Physical Review A}

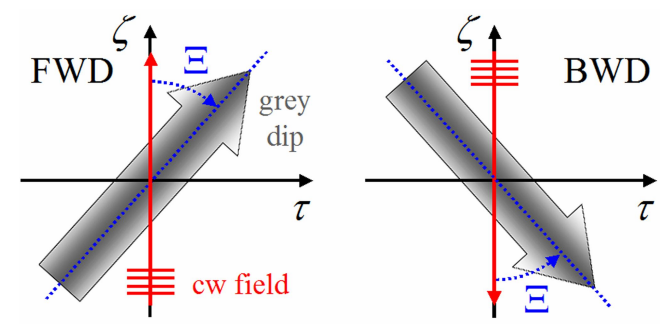

FIG. 4. (Color online) Schematic diagram illustrating the geometry of solution (17), and in particular the distinction between pulses propagating in the forward (FWD) and backward (BWD) longitudinal directions. In the $(\tau, \zeta)$ plane, the minimum of the grey dip travels along the line $\tau-V_{0} \zeta=0$ and $\tau+V_{0} \zeta=0$, respectively, where the intrinsic velocity $V_{0}$ is given by Eq. (16a). The trajectory makes an angle $\Xi$ relative to the longitudinal axis, where $\tan \Xi=V_{0}$. 


\section{Figure 5}

\section{J. M. Christian et al.}

\section{Physical Review A}

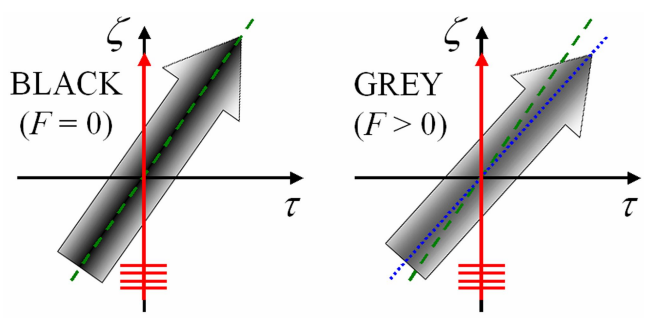

FIG. 5. (Color online) Schematic diagram illustrating the distinction between forward-propagating black and grey dark solitons. The black soliton (those solutions with $F=0$ ) has an intrinsic velocity $V_{0 \mathrm{~b}}$ [given by Eq. (16b)], while its more general grey counterpart (here, a solution with $F>0$ ) has an intrinsic velocity $V_{0}$ [where $\left.V_{0}(F>0)>V_{0 b}\right]$. 


\section{Figure 6}

\section{J. M. Christian et al.}

\section{Physical Review A}
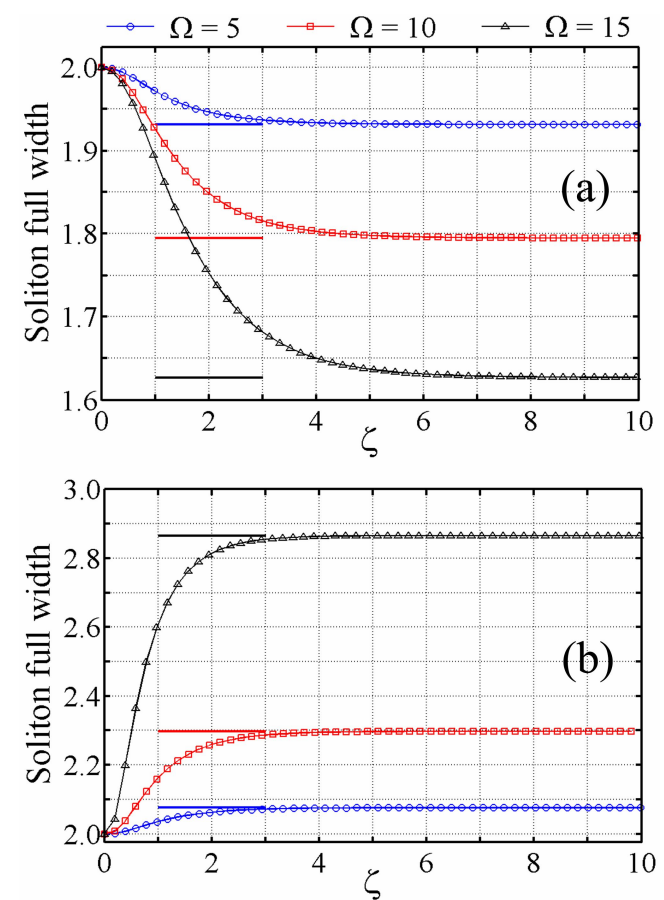

FIG. 6. (Color online) Self-reshaping of perturbed black $(F=0)$ pulses (28) toward an exact soliton with (a) $\kappa$ $=+10^{-3}$ (hyperbolic scenario) and (b) $\kappa=-10^{-3}$ (elliptic scenario - note that the qualitative features of these curves are similar to those in Ref. [23] for Helmholtz spatial dark solitons). Solid bars denote theoretical predictions of the asymptotic full-width $\Delta \tau_{\infty}$. Other parameters: $\rho_{0}=1.0$ and $\alpha=1.0$; note that the cw background of each pulse has no long-wave MI since $s=-1$. 